\title{
MKK7 is an essential component of the JNK signal transduction pathway activated by proinflammatory cytokines
}

\author{
Cathy Tournier, ${ }^{1,4}$ Chen Dong, ${ }^{2,5}$ Tod K. Turner, ${ }^{3}$ Stephen N. Jones, ${ }^{3}$ Richard A. Flavell, ${ }^{2}$ \\ and Roger J. Davis ${ }^{1,6}$

\begin{abstract}
${ }^{1}$ Howard Hughes Medical Institute, Program in Molecular Medicine, Department of Biochemistry and Molecular Biology, University of Massachusetts Medical School, Worcester, Massachusetts 01605, USA; ${ }^{2}$ Howard Hughes Medical Institute, Section of Immunobiology, Yale University School of Medicine, New Haven, Connecticut 06520, USA; ${ }^{3}$ Department of Cell Biology, University of Massachusetts Medical School, Worcester, Massachusetts 01605, USA
\end{abstract}

\begin{abstract}
Mitogen-activated protein kinases (MAPK) are activated by phosphorylation on Thr and Tyr by MAPK kinases. Two MAPK kinases (MKK4 and MKK7) can activate the c-Jun $\mathrm{NH}_{2}$-terminal kinase (JNK) group of MAPK in vitro. JNK is phosphorylated preferentially on Tyr by MKK4 and on Thr by MKK7. Targeted gene-disruption studies in mice were performed to examine the role of MKK4 and MKK7 in vivo. Simultaneous disruption of the $M k k 4$ and $M k k 7$ genes was required to block JNK activation caused by exposure of cells to environmental stress. In contrast, disruption of the $M k k 7$ gene alone was sufficient to prevent JNK activation caused by proinflammatory cytokines. These data demonstrate that MKK4 and MKK7 serve different functions in the JNK signal transduction pathway.
\end{abstract}

[Key Words: Map kinase; tumor necrosis factor; interleukin-1; JNK; MKK4; MKK7]

Received February 14, 2001; revised version accepted April 2, 2001.

The mitogen-activated protein kinases (MAPKs) represent an evolutionarily conserved signaling mechanism that is used by cells to respond to changes in their environment (Schaeffer and Weber 1999). The activation of MAPK is mediated by dual phosphorylation on a ThrXaa-Tyr motif located in the kinase activation loop. This phosphorylation is increased in stimulated cells by members of a group of MAPK kinases. These enzymes have dual substrate specificity and can phosphorylate both Thr and Tyr. Studies of a large number of MAPKs demonstrate that this mechanism of activation is conserved in many organisms, including plants, yeast, nematodes, insects, and mammals.

Two different MAPK kinases (MKK4 and MKK7) are implicated in the activation of the c-Jun $\mathrm{NH}_{2}$-terminal kinase (JNK) group of MAPK (Davis 2000). The presence of two MAPK kinases in a single MAPK signaling module is striking because genetic analysis indicates only a single MAPK kinase in each of the MAPK signaling modules of yeast (Schaeffer and Weber 1999). However, the organization of the JNK signaling pathway is similar to

Present addresses: ${ }^{4}$ School of Biological Sciences, University of Manchester, Manchester M13 9PT, UK; ${ }^{5}$ Department of Immunology, University of Washington, Seattle, WA 98195, USA.

${ }^{6}$ Corresponding author.

E-MAIL Roger.Davis@Umassmed.Edu; FAX (508) 856-3210.

Article and publication are at http://www.genesdev.org/cgi/doi/10.1101/ gad. 888501 . that of other mammalian MAPK modules. Thus, the ERK group of MAPK is activated by MKK1/MKK2 and the p38 group of MAPK is activated by MKK3/MKK6 (Schaeffer and Weber 1999). The presence of two MAPK kinases is therefore a common feature of the organization of mammalian MAPK signaling modules. The functional significance of the dual MAPK kinases found in mammalian MAPK signaling pathways is unclear.

Studies of mice demonstrate that both the Mkk4 (Yang et al. 1997; Ganiatsas et al. 1998; Nishina et al. 1999) and Mkk7 genes (Dong et al. 2000) are required for embryonic viability. This observation indicates that the MKK4 and MKK7 protein kinases serve nonredundant functions in vivo. Genetic analysis of the Mkk4 (Han et al. 1998) and Mkk7 (Glise et al. 1995) genes in Drosophila supports this conclusion. However, the molecular basis for the difference in signaling by MKK4 and MKK7 is unclear. Differences in the expression pattern of MKK4 and MKK7 in tissues may be a contributing factor. Alternatively, the distinct biochemical properties of MKK4 and MKK7 may be critical for the nonredundant functions of these protein kinases in vivo.

Comparison of the biochemical properties of MKK4 and MKK7 indicates that these protein kinases have different substrate specificities. Thus, in vitro assays demonstrate that MKK4 can activate both JNK and p38 MAPK (Derijard et al. 1995; Lin et al. 1995). In contrast, MKK7 selectively activates only JNK (Holland et al. 
1997; Moriguchi et al. 1997; Tournier et al. 1997). Although both MKK4 and MKK7 are dual-specificity protein kinases that can phosphorylate JNK on Tyr and Thr, in vitro experiments indicate that these sites are phosphorylated selectively by MKK4 and MKK7, respectively (Lawler et al. 1998). Because dual phosphorylation on these sites is thought to be required for JNK activation (Derijard et al. 1994), these in vitro experiments suggest that MKK4 and MKK7 may cooperate to activate JNK. However, it is unclear whether these data obtained from in vitro experiments reflect the physiological function of MKK4 and MKK7 in vivo.

The purpose of the study described in this report was to examine the role of MKK4 and MKK7 in vivo. Our experimental approach was to examine the JNK signaling pathway in primary murine embryo fibroblasts (MEF) isolated from wild-type, $\mathrm{Mkk}^{-/-}, \mathrm{Mkk}^{-/-}$, and dual-deficient $\mathrm{Mkk}^{-/-} \mathrm{Mkk}^{-/-}$mice.

\section{Results}

Substrate specificity of the INK activators MKK4 and $M K K 7$

We examined the specificity of MKK4 and MKK7 using in vitro protein kinase assays. MKK4 phosphorylated both $\mathrm{p} 38 \alpha$ and JNK1, whereas MKK7 phosphorylated only JNK1. Phosphoamino acid analysis demonstrated that MKK4 phosphorylated p38 $\alpha$ MAPK equally on Thr and Tyr (Fig. 1A). In contrast, equal phosphorylation of JNK1 on Thr and Tyr was not observed. MKK4 phosphorylated JNK preferentially on Tyr, whereas MKK7 phosphorylated JNK1 preferentially on Thr (Fig. 1A). However, equal phosphorylation of JNK1 on Thr and Tyr was observed in assays using MKK4 plus MKK7 (Fig. 1A). Mutational analysis demonstrated that these sites of JNK1 phosphorylation correspond to $\mathrm{Thr}^{180}$ and $\mathrm{Tyr}^{182}$.

\section{Cytokines selectively stimulate the INK activator MKK7}

Studies of recombinant MKK4 and MKK7 suggest that these protein kinases may be differentially regulated (Moriguchi et al. 1997; Tournier et al. 1997, 1999). We sought to confirm this differential regulation by examining the activity of endogenous MKK4 and MKK7. These assays demonstrated that although ultraviolet (UV) radiation and the cytotoxic drug anisomycin activated endogenous MKK4 and MKK7, the inflammatory cytokines, tumor necrosis factor (TNF), and interleukin-1 (IL-1) activated only MKK7 (Fig. 1B). This differential regulation of MKK4 and MKK7 in vivo suggests that these protein kinases may cooperate to activate JNK only in response to certain stimuli. Because MKK4 and MKK7 preferentially phosphorylate one site on the JNK dual phosphorylation motif (Thr-Pro-Tyr), it is unclear how JNK is activated in the absence of cooperation between MKK4 and MKK7. One possibility is that the in vitro specificity of MKK4 and MKK7 may not reflect in

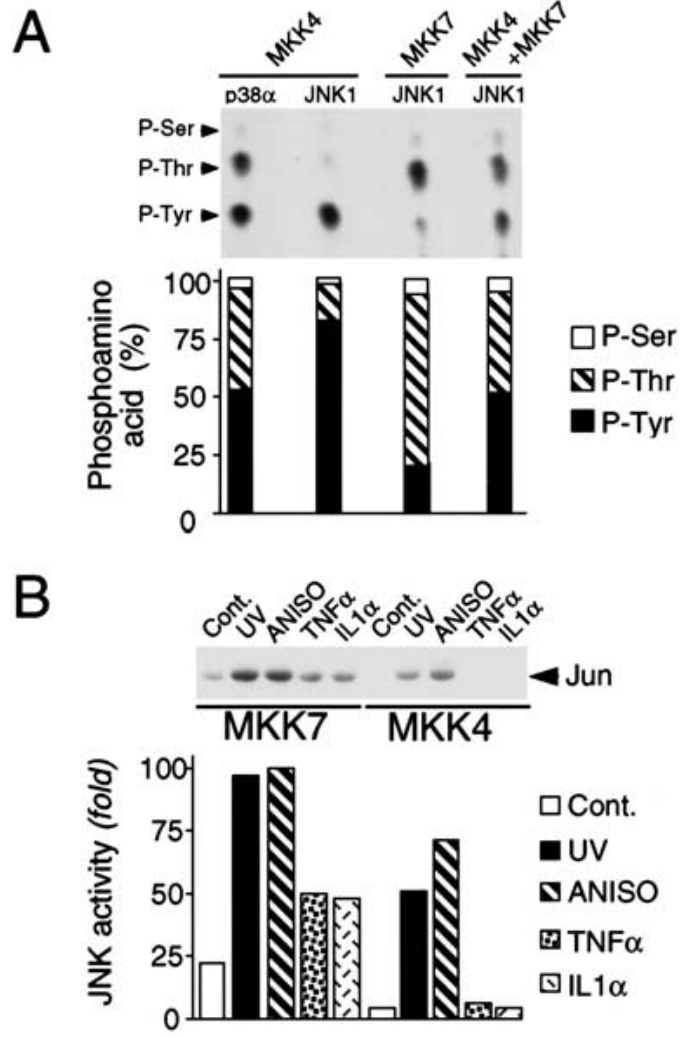

Figure 1. Biochemical characterization of MKK4 and MKK7. (A) JNK is preferentially phosphorylated on Tyr by MKK4 and on Thr by MKK7. Epitope-tagged MKK4 and MKK7 were isolated from COS cells exposed to UV-C radiation. In vitro protein kinase assays were performed using $\left[\gamma_{-}{ }^{32} \mathrm{P}\right] \mathrm{ATP}$ and purified bacterially expressed p38 $\alpha$ or JNK1 as substrates for MKK4 and MKK7. The phosphorylated JNK and p38 $\alpha$ were examined by phosphoamino acid analysis and autoradiography (upper panel). The relative phosphorylation on Thr and Tyr was examined by PhosphorImager analysis (lower panel). P-Ser, phosphothreonine; P-Thr, phosphothreonine; P-Tyr, phosphotyrosine. (B) MKK4 and MKK7 are selectively activated by extracellular stimuli. Wild-type MEF were untreated (Cont.) or treated with UV-C $\left(60 \mathrm{~J} / \mathrm{m}^{2}\right.$; UV) or anisomycin $(1 \mu \mathrm{g} / \mathrm{mL}$; ANISO) for $1 \mathrm{~h}$, or with TNF $\alpha(10 \mathrm{ng} / \mathrm{mL})$ or IL $1 \alpha(15 \mathrm{ng} / \mathrm{mL})$ for $15 \mathrm{~min}$. Endogenous MKK4 and MKK7 were immunoprecipitated using an anti-rabbit polyclonal antibody to MKK4 (K18, Santa Cruz) and a goat polyclonal antibody to MKK7 (T19, Santa Cruz), respectively. MKK activity was measured in the immune complex by a coupled protein kinase assay with JNK1 and c-Jun as the substrates. Phosphorylated c-Jun was detected after SDS-PAGE by autoradiography (upper panel) and was quantitated by PhosphorImager analysis (lower panel).

vivo specificity (e.g., interactions with scaffold proteins). A second possibility is that, unlike other MAPK, dual phosphorylation is not essential for JNK activation. To test this hypothesis, we examined the effect of mutations of the Thr and Tyr phosphorylation sites on JNK activation caused by MKK4 and MKK7 (Fig. 2). MKK4 was only able to activate wild-type JNK. In contrast, MKK7 was able to activate both wild-type JNK and a mutated JNK protein $\left(\right.$ Tyr $^{182}$ replaced with Phe). These 


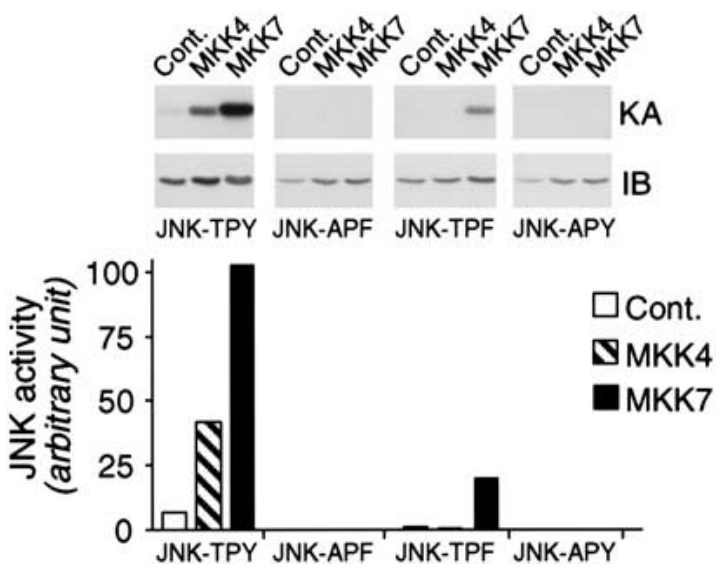

Figure 2. Phosphorylation on Thr alone causes partial JNK activation, but maximal JNK activation requires dual phosphorylation on Thr and Tyr. Cotransfection assays were performed using COS cells expressing MKK4 or MKK7 together with epitope-tagged JNK1. The effect of replacement of the dual phosphorylation site motif Thr-Pro-Tyr (TPY) with Ala-Pro-Phe (APF), Thr-Pro-Phe (TPF), or Ala-Pro-Tyr (APY) was examined. The activity of JNK1 was examined in immune complex kinase assays with the substrate c-Jun. The JNK1 proteins were detected by immunoblot analysis (IB; middle panel). JNK1 protein kinase activity was examined using the substrate c-Jun. Phosphorylated c-Jun was detected by autoradiography (KA; upper panel) and was quantitated by PhosphorImager analysis (lower panel).

data demonstrated that Tyr phosphorylation was not required for JNK activation by MKK7. However, dual phosphorylation of JNK on Thr and Tyr caused greater activation than phosphorylation on Thr alone.

\section{Isolation of MKK4- and MKK7-deficient murine embryo fibroblasts}

To test the role of the MKK4 and MKK7 protein kinases in vivo, we examined the effect of the disruption of the Mkk4 and Mkk7 genes in primary murine embryo fibroblasts (MEF). Primary cells were prepared from wild-type (WT) embryos and mutant embryos in which the Mkk4 and $M k k 7$ genes were disrupted. Immunoblot analysis demonstrated that wild-type MEF expressed both MKK4 and MKK7 (Fig. 3A). In contrast, the MKK4 and MKK7 protein kinases were not detected in $\mathrm{Mkk}^{-/-}$and $\mathrm{Mkk}^{-/-}$ MEF, respectively. Compound mutant $\mathrm{Mkk}^{4^{-/}} \mathrm{Mkk}^{-/-}$ MEF did not express MKK4 or MKK7. Control experiments demonstrated that the disruption of the $M k k 4$ and Mkk7 genes did not alter the expression of JNK or the related ERK and p38 MAPK. Proliferation assays demonstrated that the saturation density of the wild-type and mutant cells was similar, but the proliferation of the $\mathrm{Mkk}^{4^{--}}$MEF was slightly lower than wild-type cells (Fig. 3B).

MEF with targeted disruptions of all the functional Jnk genes exhibit profound defects in the response to stress, including UV radiation (Tournier et al. 2000). We there- fore examined the response of the compound mutant $M k k 4^{-/-} \mathrm{Mkk}^{-/-}$MEF to UV radiation. Exposure to UV caused a similar increase in p53 expression in wild-type, $\mathrm{Mkk}^{4^{-/-}}, \mathrm{Mkk}^{-7^{--}}$, and $\mathrm{Mkk}^{-/-} \mathrm{Mkk}^{-7^{--}} \mathrm{MEF}$ (Fig. 4A). Immunofluorescence analysis demonstrated that the UVinduced p53 protein was localized to the nucleus (Fig. 4B). Previous studies of $J n k 1^{-/-} J n k 2^{-/-}$MEF indicated that these cells have a low proliferative capacity that was associated with elevated expression of p53 and the Mdm2 inhibitor $\mathrm{p} 19^{\mathrm{ARF}}$ (Tournier et al. 2000). In contrast, the proliferative capacity of $M k k 4^{-/-} \mathrm{Mkk}^{-/-} \mathrm{MEF}$ was not reduced (Fig. 3B), and p53 expression was similar to wildtype cells (Fig. 4A,B). However, like the JNK-deficient cells (Tournier et al. 2000), the $\mathrm{Mkk}^{-/-} \mathrm{Mkk}^{-/-}$MEF did
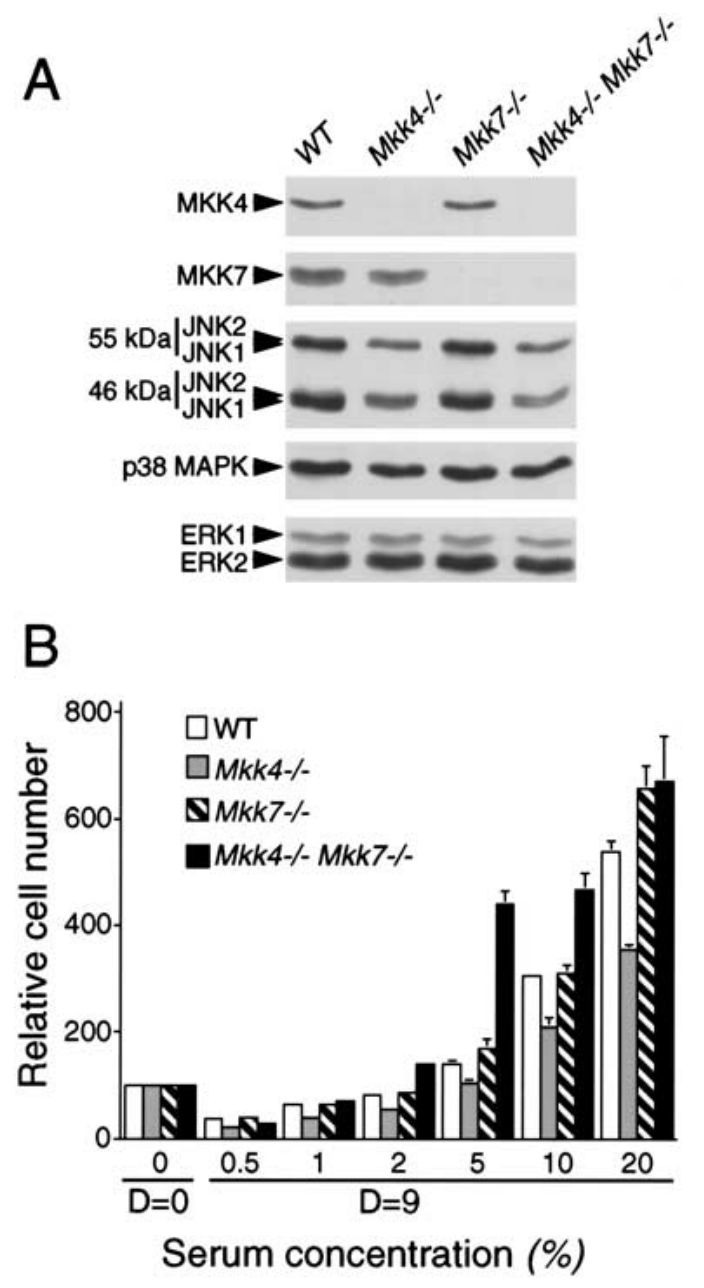

Figure 3. Isolation of MKK4- and MKK7-deficient mouse embryo fibroblasts. (A) Extracts were prepared from wild-type

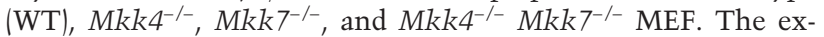
pression of MKK4, MKK7, JNK, p38 MAPK, and ERK was examined by protein immunoblot analysis. $(B)$ The saturation growth density of MEF in different concentrations of serum was examined by crystal violet staining (mean $\mathrm{OD}_{590} \pm \mathrm{SD} n=3$ ) following the addition of $1 \times 10^{4}$ cells to $20 \mathrm{~mm}$ tissue culture dishes and culture in medium supplemented with different concentrations of fetal calf serum. Relative cell numbers were measured at day $0(D=0)$ and after culture for $9 \mathrm{~d}(D=9)$. 
Tournier et al.

Figure 4. MKK4 and MKK7 are selectively required for stress-induced apopto-

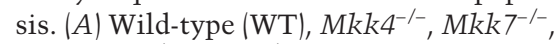
and $\mathrm{Mkk}^{-/-} \mathrm{Mkk7}^{-/-} \mathrm{MEF}$ were untreated (0) or exposed to UV-C radiation $\left(60 \mathrm{~J} / \mathrm{m}^{2}\right)$ and incubated in culture medium for 12 or $16 \mathrm{~h}$. The expression of p53, ARF, and JNK was examined by protein immunoblot analysis. (B) The subcellular distribution of p53 (green) was examined by immunofluorescence analysis. MEF were untreated (Cont.) or treated with UV-C radiation (60 $\mathrm{J} / \mathrm{m}^{2} ; \mathrm{UV}$ ) and incubated in medium with serum for $16 \mathrm{~h}$. DNA was visualized by staining with 4,6-diamidino-2-phenylindole (blue). (C) MEF were treated without and with UV-C $\left(60 \mathrm{~J} / \mathrm{m}^{2}, 15 \mathrm{~h}\right.$; UV), methymethanesulfonate $(0.5 \mu \mathrm{M}, 12 \mathrm{~h}$; MMS), or anti-Fas $(1 \mu \mathrm{g} / \mathrm{mL}$ Jo2 antibody plus $0.3 \mu \mathrm{g} /$ $\mathrm{mL}$ cycloheximide, $15 \mathrm{~h}$; FAS). The amount of apoptosis was measured by analysis of DNA fragmentation (mean $\mathrm{OD} \pm \mathrm{SD} ; n=3$ ) using the cell death detection Elisa method (Roche). (D) Extracts were prepared from cells treated without and with UV-C $\left(60 \mathrm{~J} / \mathrm{m}^{2}\right.$; UV) or taxol (5 $\mu M)$ for the indicated time. Bcl2 was detected by immunoblot analysis (IB, upper panel) and JNK activity was measured by an in vitro kinase assay. Phosphorylated c-Jun was detected by autoradioography (KA, middle panel) and was quantitated by PhosphorImager analysis (lower panel).
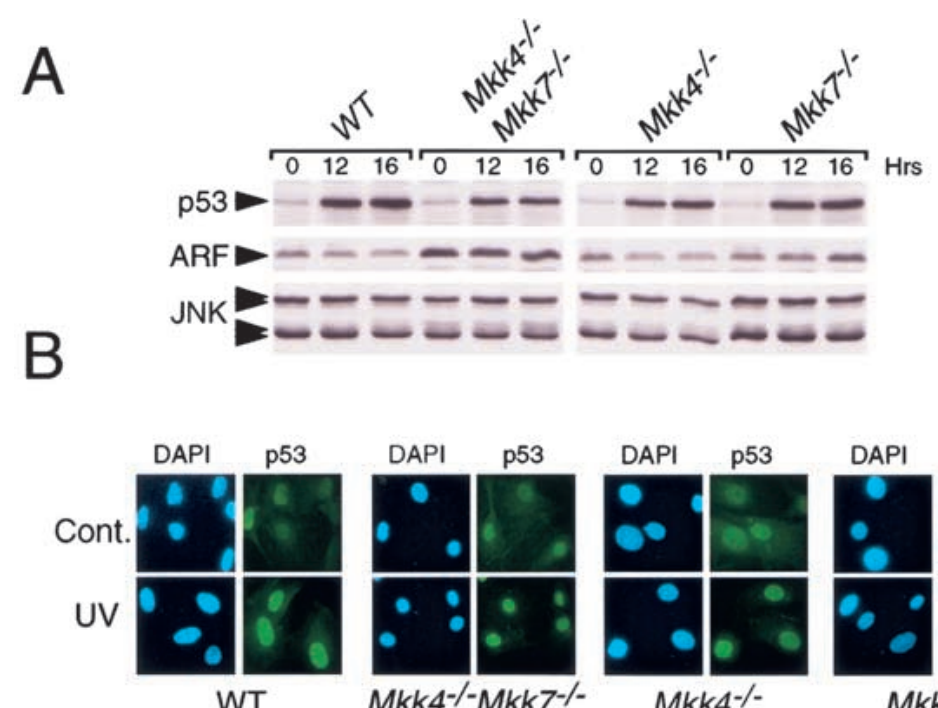

$M k k 4^{4} /-M k k 7^{-\%}$

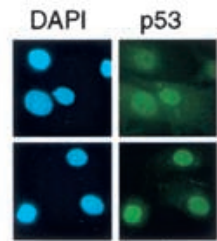

Mkk4\%

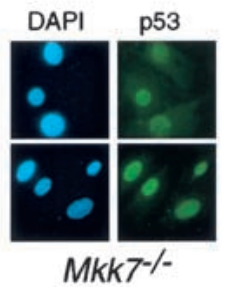

D
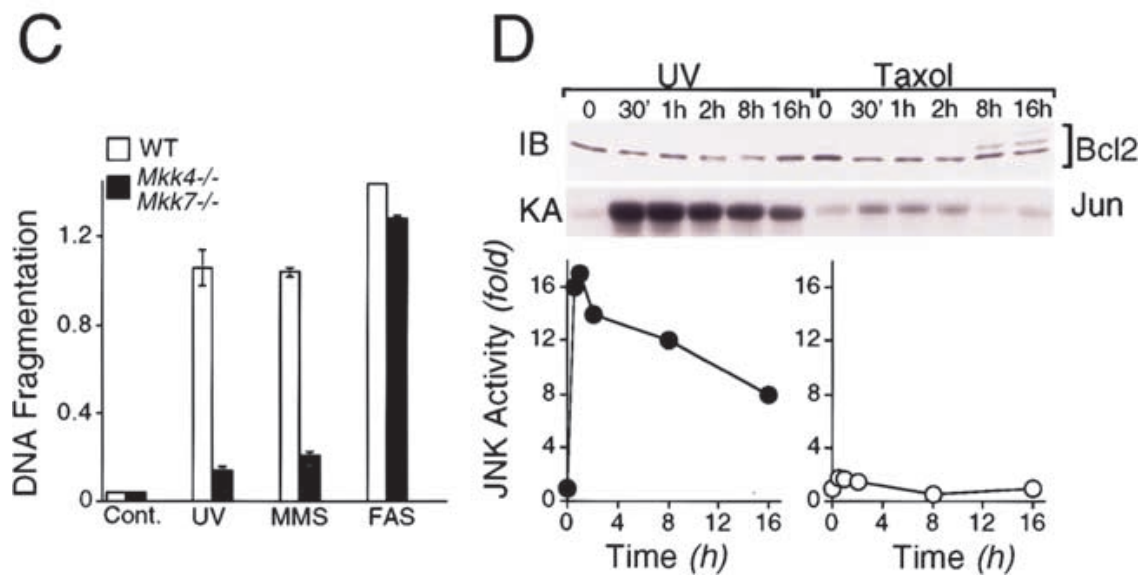

express increased amounts of p19 ${ }^{\mathrm{ARF}}$ (Fig. 4A). This increased expression of $\mathrm{p} 19^{\mathrm{ARF}}$ was not detected in $\mathrm{Mkk}^{4^{--}}$or $\mathrm{Mkk}^{-/-} \mathrm{MEF}$ (Fig. 4A). JNK-deficient MEF are selectively defective in the apoptotic response to stress (Tournier et al. 2000). Similar defects in apoptosis were detected in $\mathrm{Mkk}^{-1-} \mathrm{Mkk}^{-/-} \mathrm{MEF}$ (Fig. 4C). Thus, both JNK-deficient and JNK activator-deficient MEF were resistant to apoptosis caused by UV radiation and the alkylating agent methyl methane sulfonate, but not to activation of the Fas death receptor. It has been suggested that the effect of JNK on apoptosis may be mediated by phosphorylation of $\mathrm{Bcl} 2$ or related molecules (Haldar et al. 1995; Maundrell et al. 1997; Srivastava et al. 1999; Yamamoto et al. 1999; Kharbanda et al. 2000). However, we found no evidence that UV-activated JNK caused Bcl2 phosphorylation (Fig. 4D). In contrast, taxol caused Bcl2 phosphorylation in the absence of JNK activation (Fig. 4D). These data indicate that JNK may not be the physiologically relevant $\mathrm{Bcl} 2$ kinase. However, BH3only members of the Bcl 2 group may be targets of the JNK signaling pathway that are relevant to the apoptotic

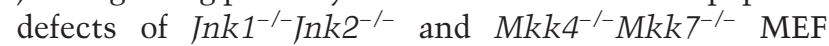
(Tournier et al. 2000).

\section{MKK7 is required for cytokine-stimulated JNK activity}

We examined JNK activity in MEF with disruptions of the Mkk4 and Mkk7 genes. The compound mutant $M k k 4^{-/-} \mathrm{Mkk}^{-/-}$MEF were severely defective in JNK activation caused by UV radiation, anisomycin, TNF, and IL-1 (Fig. 5A). In contrast, no marked changes in p38 $\alpha$ MAPK activation were detected (Fig. 5B). These data indicated that MKK4 and MKK7 represent the major activators of JNK in MEF. The small residual amount of JNK activity detected in $\mathrm{Mkk}^{-/-} \mathrm{Mkk7}^{-/-} \mathrm{MEF}$ (Fig. 5A) may indicate the presence of an additional uncharacterized JNK activator. Alternatively, the very low level of JNK activity detected may be caused by the regulated activity of a JNK phosphatase (Cavigelli et al. 1996).

Comparison of $\mathrm{Mkk}^{-/-}$and $\mathrm{Mkk}^{-/-} \mathrm{MEF}$ demonstrated similar defects in JNK activation caused by UV radiation and anisomycin. Approximately $50 \%$ of the JNK activation was lost in each of these mutant cells. Control studies using heterozygous MEF demonstrated no defects in JNK regulation, indicating that the null alleles of Mkk4 and Mkk7 were complemented by the wild-type genes (data not shown). These data suggest 
A
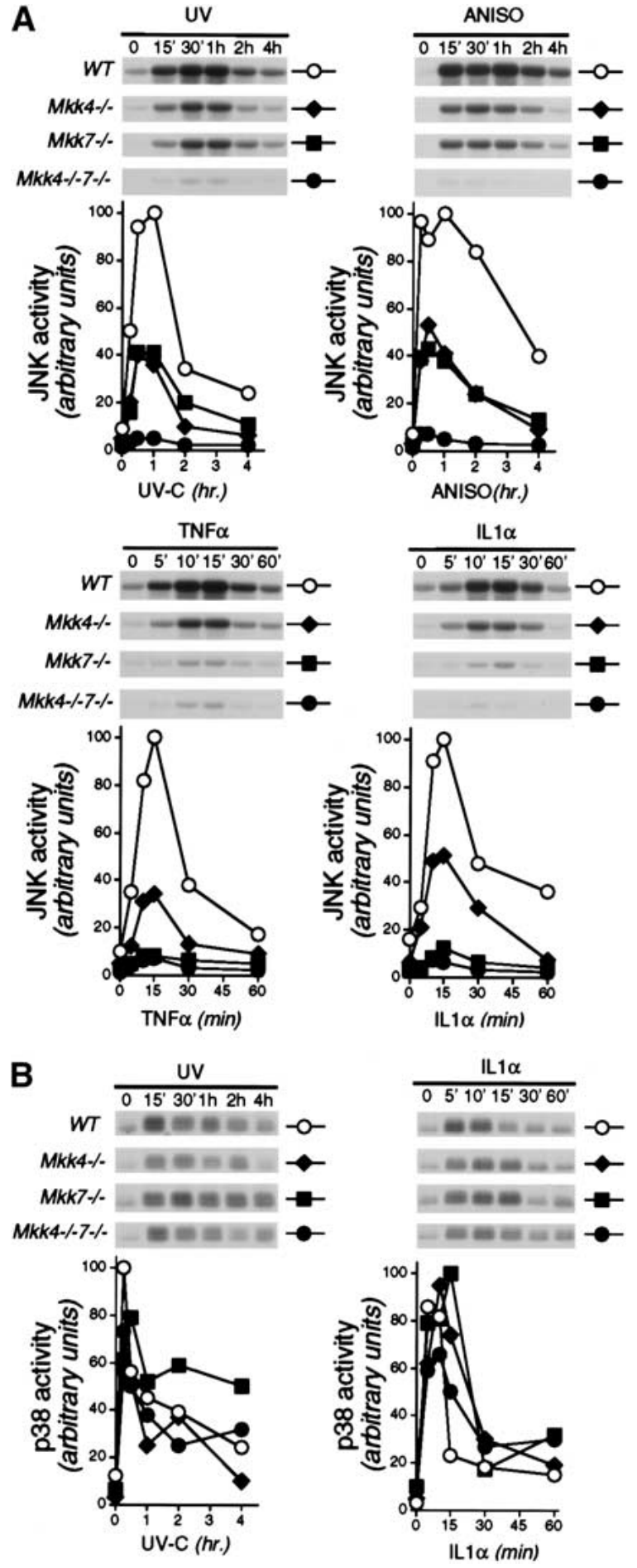

Figure 5. MKK4 and MKK7 cooperate to activate JNK in vivo. Wild-type (WT), $\mathrm{Mkk4}^{-/-}, \mathrm{Mkk7}^{-/-}$, and $\mathrm{Mkk}^{4^{--}} \mathrm{Mkk}^{-{ }^{--}} \mathrm{MEF}$ were untreated or treated with UV-C $\left(60 \mathrm{~J} / \mathrm{m}^{2} ; \mathrm{UV}\right)$, anisomycin $(1 \mu \mathrm{g} / \mathrm{mL}$; ANISO), TNF $\alpha(10 \mathrm{ng} / \mathrm{mL}$; TNF), or IL1 $\alpha$ (15 ng/mL; IL1) and then incubated for the indicated times. JNK $(A)$ and p38 MAP kinase $(B)$ activity was measured by in vitro protein kinase assay with the substrates c-Jun and ATF2, respectively. Phosphorylated c-Jun and ATF2 were detected after SDS-PAGE by autoradiography (upper), quantitated by PhosphorImager analysis (Molecular Dynamics), and presented in arbitrary units (lower). that both MKK4 and MKK7 contribute to the activation of JNK caused by UV radiation and anisomycin. This is consistent with the results of in vitro biochemical analysis of MKK4 and MKK7 specificity (Fig. 1A) and with the observation that these stress stimuli activate both MKK4 and MKK7 (Fig. 1B). In contrast, a different pattern of JNK activation was detected when these MEF were treated with the inflammatory cytokines TNF and IL-1, which activated MKK7 but not MKK4 (Fig. 1B). TNF and IL-1 did not activate JNK in $M k k 7^{-/-}$MEF (Fig. $5 \mathrm{~A})$, indicating that MKK7 is essential for JNK activation by these stimuli. However, JNK activation by TNF and IL-1 was reduced by approximately $50 \%$ in $\mathrm{Mkk}^{4^{--}} \mathrm{MEF}$ (Fig. 5A). Optimal JNK activation therefore appears to require MKK4. Because TNF and IL-1 do not activate MKK4 (Fig. 1B), these data suggest that the basal activity of MKK4 is sufficient in the presence of activated MKK7 to maximally stimulate JNK activity. No marked differences in the activation of $\mathrm{p} 38 \alpha$ MAPK were detected under these conditions (Fig. 5B).

\section{Discussion}

TNF and IL-1 are cytokines that contribute to the development of inflammatory responses. The effects of these cytokines involve several signal transduction mechanisms, including the NF-кB and AP-1 pathways. The NF$\kappa \mathrm{B}$ signal transduction pathway is mediated by the proteasomal degradation of IкB, which is initiated by the activation of an IкB kinase (Karin and Ben-Neriah 2000). The AP-1 signal transduction pathway is activated, in part, by MAPK. Indeed, the stress-activated group of MAPKs (JNK and p38) are robustly activated by TNF and IL-1 (Davis 2000). These MAPKs can induce the expression of AP-1 proteins and also increase the transcription activity of AP-1 complexes (e.g., c-Jun and ATF2) by phosphorylation (Whitmarsh and Davis 1996; Schaeffer and Weber 1999; Davis 2000).

The activation of JNK by TNF appears to be mediated by the TRAF group of adapter proteins (Liu et al. 1996; Lee et al. 1997; Natoli et al. 1997). Activation of TNF receptors leads to recruitment of the TRAF2 adapter protein (Rothe et al. 1994). Gene-disruption studies demonstrate that TRAF2 is required for JNK activation by TNF (Yeh et al. 1997). The mechansim by which TRAF2 causes JNK activation is unclear. However, TRAF2 has been reported to interact with components of the JNK signaling pathway, including the MAPK kinase kinases MEKK1 (Baud et al. 1999) and ASK1 (Nishitoh et al. 1998; Hoeflich et al. 1999). The significance of the TRAF2 interaction with MEKK1 is unclear, but TRAF2 may activate MEKK1. TRAF2 may also regulate the MAPK kinase kinase ASK1 by causing the dissociation of ASK1 from the inhibitor thioredoxin (Saitoh et al. 1998; Liu et al. 2000) and by changing ASK1 dimerization via reactive oxygen species (Gotoh and Cooper 1998). Whether MEKK1 and ASK1 serve nonredundant functions in the TRAF2 pathway and whether there are roles for additional MAPK kinase kinases in this signaling pathway is unclear. 
A role for TRAF proteins in the activation of JNK by IL-1 receptors has also been reported. The IL-1 receptor recruits TRAF6 (Cao et al. 1996), which is required for JNK activation (Lomaga et al. 1999). Like TRAF2, TRAF6 has been reported to bind MEKK1 (Baud et al. 1999), although this interaction may be mediated by the adapter protein ECSIT (Kopp et al. 1999). The ECSIT adapter protein appears to increase MEKK1 activity via proteolytic processing. TRAF6 also binds the MAPK kinase kinase TAK1 via the adapter protein TAB2 (Takaesu et al. 2000) and may lead to TAK1 activation via autophosphorylation of the T-loop (Kishimoto et al. 2000). MEKK1 and TAK1 may therefore mediate the effects of TRAF6 on activation of the JNK signal transduction pathway in cells treated with IL-1.

The relative roles of MEKK1, ASK1, and TAK1 in the cytokine receptor signal transduction pathways that cause JNK activation is unclear. Gene-disruption studies of ASK1 and TAK1 have not yet been reported. However, MEKK1 gene disruption has been examined. It was reported by one group that TNF- and IL-1-stimulated JNK activity was eliminated in $\mathrm{Mekk1}^{-/-}$ES cells (Xia et al. 2000). However, a study by an independent group using $M e k k 1^{-/-}$primary MEF isolated from MEKK1-deficient mice and Mekk1 ${ }^{-/-}$ macrophages differentiated from ES cells in vitro indicated that MEKK1 was not required for TNF- and IL-1-stimulated JNK activity (Yujiri et al. 2000). Together, these studies suggest that it is possible that MEKK1 may play a redundant role in the regulation of the JNK pathway by TNF and IL-1 in MEF. Further studies will be required to establish the roles of MEKK1, ASK1, TAK1, and other MAPK kinase kinases in the activation of JNK by cytokines.

The MAPK kinase kinases function within a MAPK signaling module composed of JNK and one of the two MAPK kinases that activate JNK (MKK4 and MKK7). The requirement of MKK4 and MKK7 for JNK activation is unclear. In this study we have examined the role of the MKK4 and MKK 7 protein kinases by targeted gene disruption and investigation of the properties of primary MEF isolated from $\mathrm{Mkk}^{-/-}, \mathrm{Mkk7}^{-/-}$, and $\mathrm{Mkk}^{-/-}$ $M k k 7^{-1}$ embryos. The results of our analysis demonstrated that both MKK4 and MKK7 are required for optimal activation of JNK in vivo. MKK4 and MKK7 appear to contribute equally to JNK activation in response to environmental stress (e.g., UV radiation). In contrast, MKK7 is essential for JNK activation by inflammatory cytokines (e.g., TNF and IL-1), and MKK4 is only required for optimal JNK activation. These data demonstrate that MKK4 and MKK7 cooperate to activate JNK in vivo. The mechanism of cooperation between MKK4 and MKK7 may be mediated by the complementary substrate specificity of these protein kinases (MKK4 and MKK7 preferentially phosphorylated JNK on Tyr ${ }^{182}$ and $\mathrm{Thr}^{180}$, respectively). Dual phosphorylation is required for optimal JNK activity, although phosphorylation on $\mathrm{Thr}^{180}$ alone is sufficient for partial JNK activation. Similar analysis of the roles of MKK1/MKK2 and MKK3/ MKK6 have not yet been reported, but it is possible that these activators of ERK and p38 MAPK, respectively, may also cooperate in vivo.
The observation that the JNK activators MKK4 and MKK7 are individually insufficient for maximal JNK activation has important functional implications for the understanding of the role of MAPK scaffold proteins. The JIP group of scaffold proteins, which bind JNK and MKK7, potentiate JNK activation by mixed-lineage protein kinases (Whitmarsh and Davis 1998). It is possible that these scaffold proteins function by tethering MAPK pathway components to increase the processivity of JNK phosphorylation on Thr and Tyr by MKK7. This mechanism would allow maximal JNK activation by MKK7 in the absence of MKK4. JNK scaffold proteins may therefore be required to create signaling modules that can be activated in response to specific stimuli without the cooperative actions of MKK4 and MKK7.

\section{Materials and methods}

Cell culture

The Mkk4 gene (Yang et al. 1997) and the Mkk7 gene (Dong et al. 2000) were disrupted by homologous recombination. MEF were prepared from E13.5 embryos and cultured in Dulbecco's modified Eagle's medium supplemented with $10 \%$ fetal calf serum (Life Technologies). All experiments were performed using cells between passage \#2 and passage \#5. Similar data were obtained in experiments using independently isolated MEF.

\section{Immunoblot analysis}

Frozen cell pellets were lysed on ice (30 $\mathrm{min}$ ) in RIPA buffer (50 $\mathrm{mM}$ Tris at $\mathrm{pH} 7.5,10 \mathrm{mM} \beta$-glycerophosphate, $5 \mathrm{mM}$ EDTA, $150 \mathrm{mM} \mathrm{NaCl}, 1 \% \mathrm{NP}-40,1 \mathrm{mM}$ phenylmethylsulfonyl fluoride, $1 \mathrm{mM}$ sodium orthovanadate, $0.4 \mathrm{U} / \mathrm{mL}$ aprotinin, and 0.4 $\mathrm{U} / \mathrm{mL}$ leupeptin). Extracts (50 $\mu \mathrm{g}$ protein) were examined by protein immunoblot analysis by probing with antibodies to MKK4 (1: 1000; Pharmingen), MKK7 (1: 1000; Zymed), JNK (1 : 2000; Pharmingen), p38 MAPK (1: 1000; Santa Cruz), ERK (1:4000; Santa Cruz), p53 (1:2000; Calbiochem), ARF (1:2000; Novus Biologicals), Bcl2 (1:1000; Pharmingen), and Flag epitope (1:4000; Sigma). Immunecomplexes were detected by enhanced chemiluminescence (Kirkegaard \& Perry).

\section{Immunofluorescence analysis}

MEF were grown on glass coverslips, fixed and permeabilized with $100 \%$ methanol at $-20^{\circ} \mathrm{C}(10 \mathrm{~min})$, and processed for immunofluorescence microscopy. The primary antibody was sheep polyclonal antibody to p53 (1:500; Calbiochem). The immune complexes were detected with fluorescein-conjugated anti-sheep Ig antibody ( $1: 200$; Jackson ImmunoResearch). Nuclei were stained ( $1 \mathrm{~min}$ ) with 4,6-diamidino-2-phenylindole (1:10,000; Molecular Probes). Fluorescence microscopy was performed with a Zeiss Axioplan microscope.

\section{Protein kinase assays}

MAPK activity (Raingeaud et al. 1995) and MAPKK activity (Tournier et al. 1999) were measured by in vitro protein kinase assays. Phosphoamino acid analysis was performed by partial acid hydrolysis and thin layer electrophoresis (Alvarez et al. 1991).

\section{Acknowledgments}

We thank Kathy Gemme for expert administrative assistance. R.A. Flavell and R.J. Davis are investigators of the Howard 
Hughes Medical Institute. This study was supported, in part, by a grant from the National Cancer Institute.

The publication costs of this article were defrayed in part by payment of page charges. This article must therefore be hereby marked "advertisement" in accordance with 18 USC section 1734 solely to indicate this fact.

\section{References}

Alvarez, E., Northwood, I.C., Gonzalez, F.A., Latour, D.A., Seth, A., Abate, C., Curran, T., and Davis, R.J. 1991. Pro-Leu-Ser/ Thr-Pro is a consensus primary sequence for substrate protein phosphorylation. Characterization of the phosphorylation of c-Myc and c-Jun proteins by an epidermal growth factor receptor threonine 669 protein kinase. J. Biol. Chem. 266: 15277-15285.

Baud, V., Liu, Z.G., Bennett, B., Suzuki, N., Xia, Y., and Karin, M. 1999. Signaling by proinflammatory cytokines: Oligomerization of TRAF2 and TRAF6 is sufficient for JNK and IKK activation and target gene induction via an amino-terminal effector domain. Genes \& Dev. 13: 1297-1308.

Cao, Z., Xiong, J., Takeuchi, M., Kurama, T., and Goeddel, D.V. 1996. TRAF6 is a signal transducer for interleukin-1. Nature 383: 443-446.

Cavigelli, M., Li, W.W., Lin, A., Su, B., Yoshioka, K., and Karin, M. 1996. The tumor promoter arsenite stimulates AP-1 activity by inhibiting a JNK phosphatase. EMBO J. 15: 62696279.

Davis, R.J. 2000. Signal transduction by the JNK group of MAP kinases. Cell 103: 239-252.

Derijard, B., Hibi, M., Wu, I.H., Barrett, T., Su, B., Deng, T., Karin, M., and Davis, R.J. 1994. JNK1: A protein kinase stimulated by UV light and Ha-Ras that binds and phosphorylates the c-Jun activation domain. Cell 76: 10251037.

Derijard, B., Raingeaud, J., Barrett, T., Wu, I.H., Han, J., Ulevitch, R.J., and Davis, R.J. 1995. Independent human MAPkinase signal transduction pathways defined by MEK and MKK isoforms. Science 267: 682-685.

Dong, C., Yang, D.D., Tournier, C., Whitmarsh, A.J., Xu, J., Davis, R.J., and Flavell, R.A. 2000. JNK is required for effector T-cell function but not for T-cell activation. Nature 405: 91-94.

Ganiatsas, S., Kwee, L., Fujiwara, Y., Perkins, A., Ikeda, T., Labow, M.A., and Zon, L.I. 1998. SEK1 deficiency reveals mitogen-activated protein kinase cascade crossregulation and leads to abnormal hepatogenesis. Proc. Natl. Acad. Sci. 95: 6881-6886.

Glise, B., Bourbon, H., and Noselli, S. 1995. hemipterous encodes a novel Drosophila MAP kinase kinase, required for epithelial cell sheet movement. Cell 83: 451-461.

Gotoh, Y. and Cooper, J.A. 1998. Reactive oxygen species- and dimerization-induced activation of apoptosis signal-regulating kinase 1 in tumor necrosis factor- $\alpha$ signal transduction. J. Biol. Chem. 273: 17477-17482.

Haldar, S., Jena, N., and Croce, C.M. 1995. Inactivation of Bcl-2 by phosphorylation. Proc. Natl. Acad. Sci. 92: 4507-4511.

Han, Z.S., Enslen, H., Hu, X., Meng, X., Wu, I.H., Barrett, T., Davis, R.J., and Ip, Y.T. 1998. A conserved p38 mitogenactivated protein kinase pathway regulates Drosophila immunity gene expression. Mol. Cell Biol. 18: 3527-3539.

Hoeflich, K.P., Yeh, W.C., Yao, Z., Mak, T.W., and Woodgett, J.R. 1999. Mediation of TNF receptor-associated factor effector functions by apoptosis signal-regulating kinase-1 (ASK1). Oncogene 18: 5814-5820.
Holland, P.M., Suzanne, M., Campbell, J.S., Noselli, S., and Cooper, J.A. 1997. MKK7 is a stress-activated mitogen-activated protein kinase kinase functionally related to hemipterous. J. Biol. Chem. 272: 24994-24998.

Karin, M. and Ben-Neriah, Y. 2000. Phosphorylation meets ubiquitination: The control of NF-кB activity. Annu. Rev. Immunol. 18: 621-663.

Kharbanda, S., Saxena, S., Yoshida, K., Pandey, P., Kaneki, M., Wang, Q., Cheng, K., Chen, Y.N., Campbell, A., Sudha, T., et al. 2000. Translocation of SAPK/JNK to mitochondria and interaction with $\mathrm{Bcl}-\mathrm{x}(\mathrm{L})$ in response to DNA damage. J. Biol. Chem. 275: 322-327.

Kishimoto, K., Matsumoto, K., and Ninomiya-Tsuji, J. 2000. TAK1 mitogen-activated protein kinase kinase kinase is activated by autophosphorylation within its activation loop. $J$. Biol. Chem. 275: 7359-7364.

Kopp, E., Medzhitov, R., Carothers, J., Xiao, C., Douglas, I., Janeway, C.A., and Ghosh, S. 1999. ECSIT is an evolutionarily conserved intermediate in the Toll/IL-1 signal transduction pathway. Genes \& Dev. 13: 2059-2071.

Lawler, S., Fleming, Y., Goedert, M., and Cohen, P. 1998. Synergistic activation of SAPK1/JNK1 by two MAP kinase kinases in vitro. Curr. Biol. 8: 1387-1390.

Lee, S.Y., Reichlin, A., Santana, A., Sokol, K.A., Nussenzweig, M.C., and Choi, Y. 1997. TRAF2 is essential for JNK but not $\mathrm{NF}-\kappa \mathrm{B}$ activation and regulates lymphocyte proliferation and survival. Immunity 7: 703-713.

Lin, A., Minden, A., Martinetto, H., Claret, F.X., Lange-Carter, C., Mercurio, F., Johnson, G.L., and Karin, M. 1995. Identification of a dual specificity kinase that activates the Jun kinases and p38-Mpk2. Science 268: 286-290.

Liu, Z.G., Hsu, H., Goeddel, D.V., and Karin, M. 1996. Dissection of TNF receptor 1 effector functions: JNK activation is not linked to apoptosis while NF- $\mathrm{B}$ activation prevents cell death. Cell 87: 565-576.

Liu, H., Nishitoh, H., Ichijo, H., and Kyriakis, J.M. 2000. Activation of apoptosis signal-regulating kinase 1 (ASK1) by tumor necrosis factor receptor-associated factor 2 requires prior dissociation of the ASK1 inhibitor thioredoxin. Mol. Cell Biol. 20: 2198-2208.

Lomaga, M.A., Yeh, W.C., Sarosi, I., Duncan, G.S., Furlonger, C., Ho, A., Morony, S., Capparelli, C., Van, G., Kaufman, S., et al. 1999. TRAF6 deficiency results in osteopetrosis and defective interleukin-1, CD40, and LPS signaling. Genes \& Dev. 13: 1015-1024.

Maundrell, K., Antonsson, B., Magnenat, E., Camps, M., Muda, M., Chabert, C., Gillieron, C., Boschert, U., Vial-Knecht, E., Martinou, J.C., et al. 1997. Bcl-2 undergoes phosphorylation by c-Jun $\mathrm{N}$-terminal kinase/stress-activated protein kinases in the presence of the constitutively active GTP-binding protein Rac1. J. Biol. Chem. 272: 25238-25242.

Moriguchi, T., Toyoshima, F., Masuyama, N., Hanafusa, H., Gotoh, Y., and Nishida, E. 1997. A novel SAPK/JNK kinase, MKK7, stimulated by TNF $\alpha$ and cellular stresses. EMBO J. 16: 7045-7053.

Natoli, G., Costanzo, A., Ianni, A., Templeton, D.J., Woodgett, J.R., Balsano, C., and Levrero, M. 1997. Activation of SAPK/ JNK by TNF receptor 1 through a noncytotoxic TRAF2-dependent pathway. Science 275: 200-203.

Nishina, H., Vaz, C., Billia, P., Nghiem, M., Sasaki, T., De la Pompa, J.L., Furlonger, K., Paige, C., Hui, C., Fischer, K.D., et al. 1999. Defective liver formation and liver cell apoptosis in mice lacking the stress signaling kinase SEK1/MKK4. Development 126: 505-516.

Nishitoh, H., Saitoh, M., Mochida, Y., Takeda, K., Nakano, H., Rothe, M., Miyazono, K., and Ichijo, H. 1998. ASK1 is essen- 
tial for JNK/SAPK activation by TRAF2. Mol. Cell 2: 389 395.

Raingeaud, J., Gupta, S., Rogers, J.S., Dickens, M., Han, J., Ulevitch, R.J., and Davis, R.J. 1995. Pro-inflammatory cytokines and environmental stress cause p38 mitogen-activated protein kinase activation by dual phosphorylation on tyrosine and threonine. J. Biol. Chem. 270: 7420-7426.

Rothe, M., Wong, S.C., Henzel, W.J., and Goeddel, D.V. 1994. A novel family of putative signal transducers associated with the cytoplasmic domain of the $75 \mathrm{kDa}$ tumor necrosis factor receptor. Cell 78: 681-692.

Saitoh, M., Nishitoh, H., Fujii, M., Takeda, K., Tobiume, K., Sawada, Y., Kawabata, M., Miyazono, K., and Ichijo, H. 1998. Mammalian thioredoxin is a direct inhibitor of apoptosis signal-regulating kinase (ASK) 1. EMBO J. 17: 2596-2606.

Schaeffer, H.J. and Weber, M.J. 1999. Mitogen-activated protein kinases: Specific messages from ubiquitous messengers. Mol. Cell Biol. 19: 2435-2444.

Srivastava, R.K., Mi, Q.S., Hardwick, J.M., and Longo, D.L. 1999. Deletion of the loop region of Bcl-2 completely blocks paclitaxel-induced apoptosis. Proc. Natl. Acad. Sci. 96: 3775-3780.

Takaesu, G., Kishida, S., Hiyama, A., Yamaguchi, K., Shibuya, H., Irie, K., Ninomiya-Tsuji, J., and Matsumoto, K. 2000 TAB2, a novel adaptor protein, mediates activation of TAK1 MAPKKK by linking TAK1 to TRAF6 in the IL-1 signal transduction pathway. Mol. Cell 5: 649-658.

Tournier, C., Whitmarsh, A.J., Cavanagh, J., Barrett, T., and Davis, R.J. 1997. Mitogen-activated protein kinase kinase 7 is an activator of the c-Jun $\mathrm{NH}_{2}$-terminal kinase. Proc. Natl. Acad. Sci. 94: 7337-7342.

- 1999. The MKK7 gene encodes a group of c-Jun $\mathrm{NH}_{2}$ terminal kinase kinases. Mol. Cell Biol. 19: 1569-1581.

Tournier, C., Hess, P., Yang, D.D., Xu, J., Turner, T.K., Nimnual, A., Bar-Sagi, D., Jones, S.N., Flavell, R.A., and Davis, R.J. 2000. Requirement of JNK for stress-induced activation of the cytochrome c-mediated death pathway. Science 288: 870-874.

Whitmarsh, A.J. and Davis, R.J. 1996. Transcription factor AP-1 regulation by mitogen-activated protein kinase signal transduction pathways. J. Mol. Med. 74: 589-607.

- 1998. Structural organization of MAP-kinase signaling modules by scaffold proteins in yeast and mammals. Trends Biochem. Sci. 23: 481-485.

Xia, Y., Makris, C., Su, B., Li, E., Yang, J., Nemerow, G.R., and Karin, M. 2000. MEK kinase 1 is critically required for c-Jun $\mathrm{N}$-terminal kinase activation by proinflammatory stimuli and growth factor-induced cell migration. Proc. Nat1. Acad. Sci. 97: 5243-5248.

Yamamoto, K., Ichijo, H., and Korsmeyer, S.J. 1999. BCL-2 Is phosphorylated and inactivated by an ASK1/Jun N-terminal protein kinase pathway normally activated at G(2)/M. Mol. Cell Biol. 19: 8469-8478.

Yang, D., Tournier, C., Wysk, M., Lu, H.T., Xu, J., Davis, R.J., and Flavell, R.A. 1997. Targeted disruption of the MKK4 gene causes embryonic death, inhibition of c-Jun $\mathrm{NH}_{2}$-terminal kinase activation, and defects in AP-1 transcriptional activity. Proc. Natl. Acad. Sci. 94: 3004-3009.

Yeh, W.C., Shahinian, A., Speiser, D., Kraunus, J., Billia, F., Wakeham, A., de la Pompa, J.L., Ferrick, D., Hum, B., Iscove, N., et al. 1997. Early lethality, functional NF-кB activation, and increased sensitivity to TNF-induced cell death in TRAF2-deficient mice. Immunity 7: 715-725.

Yujiri, T., Ware, M., Widmann, C., Oyer, R., Russell, D., Chan, E., Zaitsu, Y., Clarke, P., Tyler, K., Oka, Y., et al. 2000. MEK kinase 1 gene disruption alters cell migration and c-Jun $\mathrm{NH}_{2}$ - terminal kinase regulation but does not cause a measurable defect in NF-кB activation. Proc. Natl. Acad. Sci. 97: 72727277. 


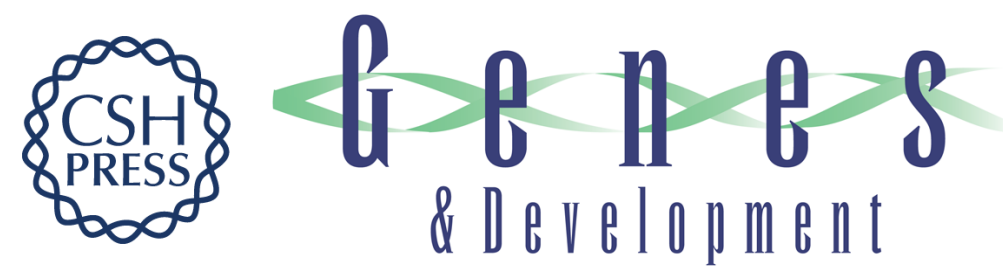

\section{MKK7 is an essential component of the JNK signal transduction pathway activated by proinflammatory cytokines}

Cathy Tournier, Chen Dong, Tod K. Turner, et al.

Genes Dev. 2001, 15:

Access the most recent version at doi:10.1101/gad.888501

References This article cites 46 articles, 29 of which can be accessed free at: http://genesdev.cshlp.org/content/15/11/1419.full.html\#ref-list-1

License

Email Alerting

Receive free email alerts when new articles cite this article - sign up in the box at the top Service right corner of the article or click here.

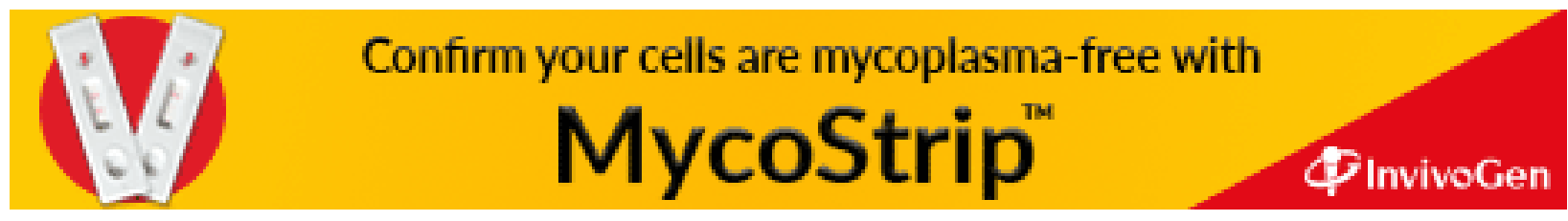

